the ASME 2016 International Design Engineering Technical Conferences \& Computers and Information in Engineering Conference IDETC/CIE 2016

August 21-242016Charlotte, North CarolinaUSA

\title{
IDETC2016/59804
}

\section{OPERATION MODES COMPARISON OF A RECONFIGURABLE 3-PRS PARALLEL MANIPULATOR BASED ON KINEMATIC PERFORMANCE}

\author{
Siddharth Maraje ${ }^{1}$, Latifah Nurahmi ${ }^{2}$, Stéphane Caro ${ }^{3 *}$ \\ 1 École Centrale Nantes, IRCCyN, 1, rue de la Noë, 44321 Nantes, France \\ ${ }^{2}$ Mechanical Engineering, Institut Teknologi Sepuluh Nopember, Surabaya, Indonesia \\ ${ }^{3}$ CNRS, IRCCyN, 1, rue de la Noë, 44321 Nantes, France \\ Emails: \{siddharth.maraje, latifah.nurahmi\}@gmail.com, stephane.caro@irccyn.ec-nantes.fr
}

\begin{abstract}
The 3-PRS parallel manipulator with different arrangements of prismatic joints is called a reconfigurable 3-PRS parallel manipulator in this paper. The three prismatic joints in PRS limbs are attached to the base with an angle $\alpha$ between the horizontal plane of the base and their directions. Based on [1], the manipulator has identical operation modes, namely $x_{0}=0$ and $x_{3}=0$ for any value of $\alpha$. Accordingly, this paper presents in more details the performance evaluation of these operation modes by using the output transmission index (OTI) and the constraint transmission index $(C T I)$. The OTI and CTI determine the force transmission efficiency and the constraining ability of the manipulators, respectively.

Initially, the determination of the number and types of operation modes of the 3-PRS parallel manipulator is recalled. The computation is carried out by using an algebraic approach, namely the Study kinematic mapping. In each operation mode, the actuation wrenches and the constraint wrenches are obtained based on the Screw theory. Then, the OTI and CTI are traced in the orientation workspace of the manipulator for different values of angle $\alpha$. Furthermore, the singularity conditions are analysed corresponding to the values of OTI and CTI.
\end{abstract}

\section{INTRODUCTION}

The 3-PRS parallel manipulator ( $\mathrm{P}, \mathrm{R}$ and $\mathrm{S}$ denote prismatic, revolute and spherical joints, respectively) is a lower-

*Address all correspondence to this author. mobility $(d o f)$ parallel manipulator $(n<6)$. This manipulator is called reconfigurable since the arrangement of the prismatic joints can be changed by varying $\alpha$, which is the angle between the directions of prismatic joint axes and the horizontal plane of the base. Reconfigurable manipulators are of special interest as the workspace of these manipulators can be significantly increased by varying the architecture of their reconfigurable base. In [2], the operation modes of a 4-RUU parallel manipulator were analyzed. It is shown that the 4-RUU parallel manipulator is able to perform 3 different operation modes. As a consequence, the manipulator is said to be reconfigurable.

The detailed kinematic analysis of a reconfigurable 3-PRS parallel manipulator was presented by $\mathrm{Li}$ et al. in [3]. These designs are considered to be of the same type as they can be represented by using the same kinematic equations. Over the years this manipulator has been extensively studied to improve its utility. One of such early developments was a machining tool head called Sprint Z3 developed by DS technologies in Germany [4]. The peculiarity of this mechanism is that the axes of all the three prismatic joints are arranged vertically. This arrangement is named as 3-P $\mathrm{P}_{\mathrm{V}} \mathrm{RS}$ by Liu and Bonev in [5] where they studied the relation between the accuracy of the $3-\mathrm{P}_{\mathrm{V}} \mathrm{RS}$ and its tilt angle. They showed that there exists an inverse relation between accuracy and tilt angle. Fan et al. identified the critical parameters (positioning accuracy of the prismatic joints and the tool length) that affect the positions and orientations of the platform [6]. Gan et al. presented a metamorphic parallel mechanism (MPM) capable of switching its motion between pure translation (3T) and 
pure rotation (3R) [7]. Kinematic analysis of the mechanism was performed covering both $3 \mathrm{~T}$ and $3 \mathrm{R}$ motions. In [8] they presented a unified kinematics and optimal design of a 3rRPS MPM with a reconfigurable revolute joint.

Kinematic analysis of the 3-RPS cube parallel manipulator, which belongs to the family of three dof parallel manipulators having one operation mode, was done in [9]. Recently, Nurahmi et al. analysed the 3-PRS parallel manipulator in [1] using an algebraic approach called 'Study kinematic mapping' of the Euclidean group $S E(3)$. The mechanism was described by using a set of eight constraint equations. A primary decomposition over a set of constraint equations revealed the existence of two operation modes. Singularity conditions were also determined by calculating the determinant of the Jacobian matrix with respect to the Study parameters. Following the research conducted in [1], this paper focuses on the study of the force/motion transmission capabilities of the two operation modes of the 3-PRS parallel manipulator for different values of angle $\alpha$. Output and constraint transmission indices are computed to evaluate the force/motion transmission capability in each operation mode. Eventually, the performance of the two operation modes can be compared and analysed within the orientation workspace. Some manipulator poses are presented to visualize the effectiveness of these indices.

This paper is organised as follows: Section 2 presents a brief review on performance indices associated with output and constraint transmission indices. Section 3 presents the architecture of the manipulator and its operation modes. In section 4, the Jacobian matrix of the 3-PRS manipulator is derived based on the Screw theory. In section 5, performance comparison of the two operation modes is discussed based on the distribution of output and constraint transmission indices in the orientation workspace. Eventually, section 6 discusses some of the singular configurations of the manipulator in the two operation modes.

\section{TRANSMISSION INDICES}

Transmission indices attempt to assess the quality of force/motion transmission of a manipulator. These indices are also an alternative way to overcome the issue of nonhomogeneity in the Jacobian matrix. Several transmission indices have been proposed in the literature. Virtual coefficient proposed in [10] determines the virtual power developed by an applied wrench on the twist of the moving platform. The higher the value of the virtual coefficient, the higher the virtual power and hence the better the force transmission capability. A finite dimensionless index was proposed by Roth and Sutherland in [11] by normalising the value of virtual coefficient. The internal wrench arising due to the force transmission was termed as transmission wrench and the motion generated by this transmission wrench was named output twist screw. Moreover, they introduced the concept of a characteristic length, which is the shortest distance between the transmission wrench screw and the output twist screw, to calculate the maximum value of the virtual coefficient. Chen and Angeles suggested a generalised transmission index in [12] by introducing the concept of an application point at the centroid of the contact region in the floating pair of the output link. Thus, the calculation of the maximum value of the virtual coefficient is no longer configuration dependent. In [13], three indices were proposed to evaluate the effectiveness of the force/motion transmission of the 3-PRS parallel manipulator. These indices were also used to optimize the link lengths for obtaining better force/motion transmission capability and higher orientation capability.

Liu et al. proposed a new approach to analyse force transmission capabilities of parallel manipulators and determine their closeness to singularities [12-14]. In [17] they proposed a generalized approach to calculate the maximal value of virtual coefficient for parallel manipulators. As a result, two transmission indices, namely output transmission index (OTI) and constraint transmission index (CTI) were proposed. The definitions of OTI and CTI are briefly discussed in the following sections.

\section{Output Transmission Index (OTI)}

The presence of an actuated joint in the limbs of a parallel manipulator applies an actuation wrench on to the moving platform. This wrench can be a pure force, a pure moment, or a finite-pitch wrench. Thus, OTI is defined to characterize the actuation wrench transmitted to the motion of the moving platform, as:

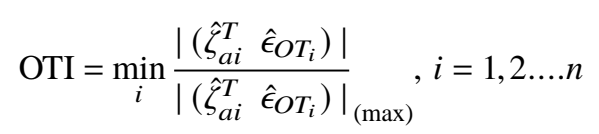

where,

$\hat{\zeta}_{a i}$ is the $i$-th actuation wrench screw produced by the $i$-th actuated joint,

$\hat{\epsilon}_{O T_{i}}$ is the $i$-th output twist, obtained by locking all the actuated joints but the $i$-th one and observing small pose variations of the moving platform.

The OTI is bounded between 0 to 1 . When the value of OTI is equal to 1 , the force transmission capability of the particular configuration is optimal. On the other hand, OTI vanishes in actuation singularity configurations.

\section{Constraint Transmission Index (CTI)}

In lower-mobility parallel manipulators, some of the motions of the moving platform are restricted due to the constraint wrenches induced by the limbs. Those wrenches can be pure forces, pure moments, and/or finite-pitch wrenches depending on the architecture of the manipulator. Therefore, CTI characterizes 
the ability of the limbs of the manipulator to constrain the motion of its moving platform. The Constraint Transmission Index (CTI) is expressed as follows:

where,

$$
\mathrm{CTI}=\min _{j} \frac{\left|\left(\hat{\zeta}_{c j}^{T} \hat{\epsilon}_{C T_{j}}\right)\right|}{\left|\left(\hat{\zeta}_{c j}^{T} \hat{\epsilon}_{C T_{j}}\right)\right|}{ }_{(\max )}, j=1,2 \ldots .(6-n)
$$

$\hat{\zeta}_{c j}$ is the $j^{t h}$ constraint wrench screw applied by the $j^{\text {th }}$ limb on the moving platform,

$\hat{\epsilon}_{C T_{j}}$ is the $j^{\text {th }}$ restricted twist screw, produced by locking all the actuated joints and releasing one characteristic constraint direction along a virtual constraint joint, and observing variations in the pose of the moving platform.

On the one hand, CTI is equal to 1 when the arrangement of the constraint wrenches is optimal, namely, the ability of the manipulator to constrain the undesirable motions of the movingplatform is the best. On the other hand, CTI is null when the manipulator reaches a constraint singularity.

\section{THE 3-PRS PARALLEL MANIPULATOR}

The architecture of the 3-PRS parallel manipulator studied in this paper is shown in Fig. 1. The plastic model of this manipulator is shown in Fig. 2. The base and the moving platform are equilateral triangles and their circumradii are in the proportion 2:1 (base:platform). Three identical limbs connect the base to the moving platform where each limb consists of a prismatic joint, a revolute joint, and a spherical joint connected in series. The origin of the base frame is located at the circumcenter (point $O$ ) of the base equilateral triangle. $A_{1}, A_{2}$ and $A_{3}$ are the three vertices of the base triangle with circumradius $h_{0}$. The prismatic joint of the $i$-th $(i=1,2,3)$ limb is arranged in such a way that it makes an angle $\alpha$ with the base plane and is directed along vector $\mathbf{p}_{i}$. The revolute joint of the $i$-th limb is located at point $B_{i}$ with its axis along vector $\mathbf{s}_{i}$. The origin of the moving frame is fixed at the circumcenter (point $P$ ) of an equilateral triangle. The moving platform is connected to the limbs through three spherical joints at points $C_{1}, C_{2}$ and $C_{3}$ and its circumradius is $h_{1}$.

The length of segment $B_{i} C_{i}$, is equal to $l$ and the segment $B_{i} C_{i}$ is perpendicular to vector $\mathbf{s}_{i}$. As a result, the segment $B_{i} C_{i}$ moves in a plane normal to $\mathbf{s}_{i}$. The motion of the manipulator depends mainly on the four design parameters $l, h_{0}, h_{1}, \alpha$ and the three joint variables $r_{1}, r_{2}, r_{3}$ directed along vectors $\mathbf{p}_{1}, \mathbf{p}_{2}$ and $\mathbf{p}_{3}$ respectively.

The coordinates of the points $B_{i}$ and $C_{i}$ are expressed in the base frame $\Sigma_{0}$ and the platform frame $\Sigma_{1}$ respectively as follows:

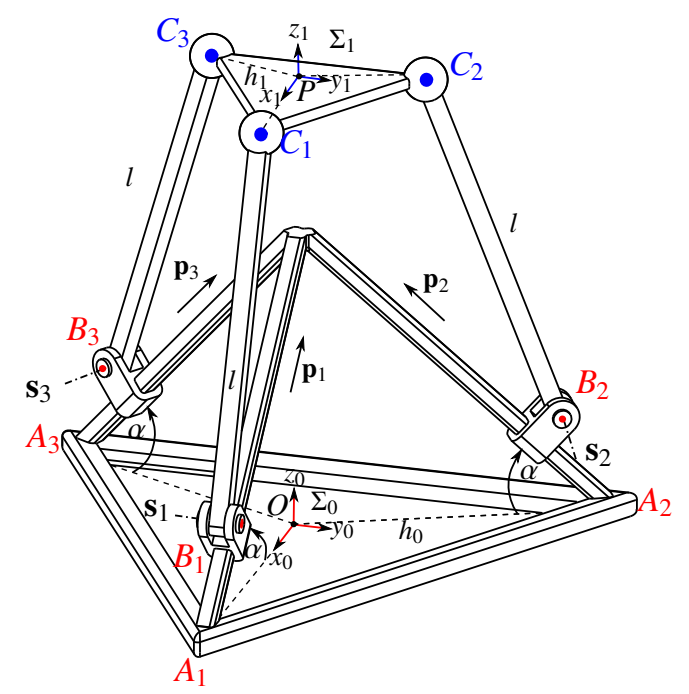

FIGURE 1: THE 3-PRS PARALLEL MANIPULATOR

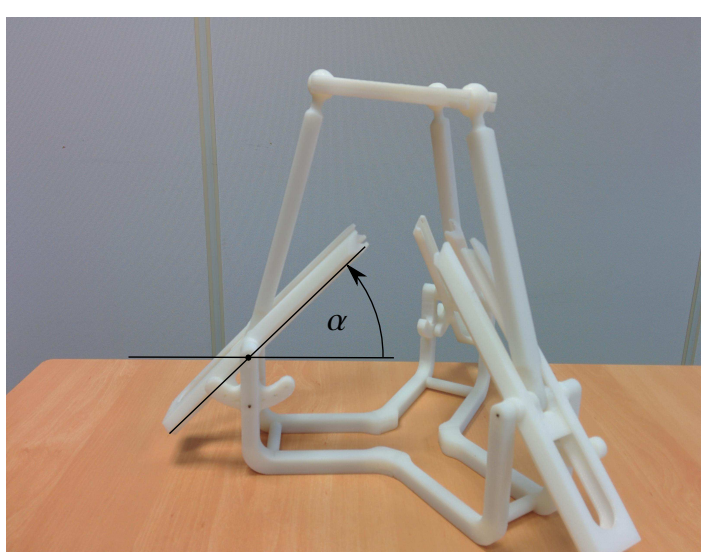

FIGURE 2: PLASTIC MODEL OF THE 3-PRS PARALLEL MANIPULATOR

$$
\begin{gathered}
\mathbf{r}_{B_{1}}^{0}=\left[1, h_{0}-r_{1} \cos (\alpha), 0, r_{1} \sin (\alpha)\right]^{T}, \\
\mathbf{r}_{B_{2}}^{0}=\left[1,-\frac{1}{2}\left(h_{0}-r_{2} \cos (\alpha)\right), \frac{\sqrt{3}}{2}\left(h_{0}-r_{2} \cos (\alpha)\right), r_{2} \sin (\alpha)\right]^{T}, \\
\mathbf{r}_{B_{3}}^{0}=\left[1,-\frac{1}{2}\left(h_{0}-r_{3} \cos (\alpha)\right),-\frac{\sqrt{3}}{2}\left(h_{0}-r_{3} \cos (\alpha)\right), r_{3} \sin (\alpha)\right]^{T}, \\
\mathbf{r}_{C_{1}}^{1}=\left[1, h_{1}, 0,0\right]^{T}, \\
\mathbf{r}_{C_{2}}^{1}=\left[1,-\frac{1}{2} h_{1}, \frac{\sqrt{3}}{2} h_{1}, 0\right]^{T}, \\
\mathbf{r}_{C_{3}}^{1}=\left[1,-\frac{1}{2} h_{1},-\frac{\sqrt{3}}{2} h_{1}, 0\right]^{T} .
\end{gathered}
$$

The Study parametrization of a spatial Euclidean transformation matrix $\mathbf{M} \in S E(3)$ is used to obtain the coordinates of points $C_{1}, C_{2}, C_{3}$ in the base frame $\Sigma_{0}$ as follows: 


$$
\mathbf{M}=\left(\begin{array}{cc}
x_{0}^{2}+x_{1}^{2}+x_{2}^{2}+x_{3}^{2} & \mathbf{0}_{3 \times 1}^{T} \\
\mathbf{M}_{T} & \mathbf{M}_{R}
\end{array}\right)
$$

where $\mathbf{M}_{T}$ and $\mathbf{M}_{R}$ are the translational and rotational parts of the transformation matrix $\mathbf{M}$, respectively and are given as:

$$
\begin{gathered}
\mathbf{M}_{T}=\left(\begin{array}{c}
2\left(-x_{0} y_{1}+x_{1} y_{0}-x_{2} y_{3}+x_{3} y_{2}\right) \\
2\left(-x_{0} y_{2}+x_{1} y_{3}+x_{2} y_{0}-x_{3} y_{1}\right) \\
2\left(-x_{0} y_{3}-x_{1} y_{2}+x_{2} y_{1}+x_{3} y_{0}\right)
\end{array}\right), \\
\mathbf{M}_{R}=\left(\begin{array}{ccc}
x_{0}^{2}+x_{1}^{2}-x_{2}^{2}-x_{3}^{2} & 2\left(x_{1} x_{2}-x_{0} x_{3}\right) & 2\left(x_{1} x_{3}+x_{0} x_{2}\right) \\
2\left(x_{1} x_{2}+x_{0} x_{3}\right) & x_{0}^{2}-x_{1}^{2}+x_{2}^{2}-x_{3}^{2} & 2\left(x_{2} x_{3}-x_{0} x_{1}\right) \\
2\left(x_{1} x_{3}-x_{0} x_{2}\right) & 2\left(x_{2} x_{3}+x_{0} x_{1}\right) & x_{0}^{2}-x_{1}^{2}-x_{2}^{2}+x_{3}^{2}
\end{array}\right)
\end{gathered}
$$

The parameters $x_{0}, x_{1}, x_{2}, x_{3}, y_{0}, y_{1}, y_{2}, y_{3}$, appearing in matrix M, are called Study-parameters which enable to parametrize $S E(3)$ with dual quaternions.

\section{Operation Modes}

The operation modes of the 3-PRS manipulator were identified in [1]. For given values of the actuated joint variables, point $P$ of the moving platform has the same Cartesian coordinates in both operation modes. However, the orientations of the moving platform are different. It makes the difference between the two operation modes $x_{0}=0$ and $x_{3}=0$. The transformation matrices between the base and the moving-platform frames are determined in [1].

\section{Operation Mode $x_{0}=0$}

In this operation mode, every platform pose satisfies the condition $x_{0}=0$ and the transformation matrix between the base frame and the platform frame is expressed as:

$$
\mathbf{T}_{1}=\left(\begin{array}{cccc}
1 & 0 & 0 & 0 \\
h_{1}\left(x_{1}^{2}-x_{2}^{2}\right) & x_{1}^{2}-x_{2}^{2}-x_{3}^{2} & 2 x_{1} x_{2} & 2 x_{1} x_{3} \\
-2 h_{1} x_{1} x_{2} & 2 x_{1} x_{2} & -\left(x_{1}^{2}-x_{2}^{2}+x_{3}^{2}\right) & 2 x_{2} x_{3} \\
Z & 2 x_{1} x_{3} & 2 x_{2} x_{3} & -\left(x_{1}^{2}+x_{2}^{2}-x_{3}^{2}\right)
\end{array}\right)
$$

where, $Z=\frac{h_{1}\left(x_{1}^{2} x_{3}-x_{2}^{2} x_{3}\right)-2\left(x_{1}^{2} y_{2}+x_{2}^{2} y_{2}+x_{3}^{2} y_{2}\right)}{x_{1}}$

\section{Operation mode $x_{3}=0$}

In this operation mode, every platform pose of manipulator satisfies the condition, $x_{3}=0$. The transformation matrix between the base frame and the moving-platform frame for this operation mode is expressed as:

$$
\mathbf{T}_{2}=\left(\begin{array}{cccc}
1 & 0 & 0 & 0 \\
h_{1}\left(x_{1}^{2}-x_{2}^{2}\right) & x_{0}^{2}+x_{1}^{2}-x_{2}^{2} & 2 x_{1} x_{2} & 2 x_{0} x_{2} \\
-2 h_{1} x_{1} x_{2} & 2 x_{1} x_{2} & x_{0}^{2}-x_{1}^{2}+x_{2}^{2} & -2 x_{0} x_{1} \\
Z & -2 x_{0} x_{2} & 2 x_{0} x_{1} & x_{0}^{2}-x_{1}^{2}-x_{2}^{2}
\end{array}\right)
$$

where $Z=\frac{h_{1}\left(x_{1}^{2} x_{0}-x_{2}^{2} x_{0}\right)+2\left(x_{0}^{2} y_{1}+x_{1}^{2} y_{1}+x_{2}^{2} y_{1}\right)}{x_{2}}$

\section{JACOBIAN MATRIX OF THE 3-PRS MANIPULATOR}

By considering that the prismatic joints are the actuated ones, each limb applies one actuation force on the moving platform whose axis passes through points $B_{i}$ and $C_{i}$ of unit vector $\mathbf{u}_{i}$, as follows:

$$
\begin{aligned}
& \hat{\zeta}_{a 1}=\left(\mathbf{u}_{1}, \mathbf{r}_{C_{1}}^{0} \times \mathbf{u}_{1}\right) \\
& \hat{\zeta}_{a 2}=\left(\mathbf{u}_{2}, \mathbf{r}_{C_{2}}^{0} \times \mathbf{u}_{2}\right) \\
& \hat{\zeta}_{a 3}=\left(\mathbf{u}_{3}, \mathbf{r}_{C_{3}}^{0} \times \mathbf{u}_{3}\right)
\end{aligned}
$$

Due to the manipulator architecture, each limb applies one constraint force, which is perpendicular to the actuated prismatic joint, parallel to the axis $\mathbf{s}_{i}$ of the revolute joint passes through point $C_{i}$, written as:

$$
\begin{aligned}
& \hat{\zeta}_{c 1}=\left(\mathbf{s}_{1}, \mathbf{r}_{C_{1}}^{0} \times \mathbf{s}_{1}\right) \\
& \hat{\zeta}_{c 2}=\left(\mathbf{s}_{2}, \mathbf{r}_{C_{2}^{0}} \times \mathbf{s}_{2}\right) \\
& \hat{\zeta}_{c 3}=\left(\mathbf{s}_{3}, \mathbf{r}_{C_{3}}^{0} \times \mathbf{s}_{3}\right)
\end{aligned}
$$

In Fig.3, the actuation (blue vectors) as well as the constraint wrenches (green vectors) of the manipulator are shown.

To evaluate OTI and CTI, we need to determine the output twist screws and the constraint twist screws. In the following section, an analytical approach presented in [17] is adapted to determine output and constraint twist screws.

\section{Output and Constraint twist screws}

The constraint wrench system of the 3-PRS parallel manipulator is spanned by the three zero pitch wrenches. Due to this condition, there exist three infinite-pitch constraint twist screws along the directions parallel to the axes of revolute joints. Thus, to assess the constraining ability of the limbs, three virtual prismatic joints can be added at the center of the spherical joints, their directions being parallel to the axes of revolute joints, as represented in Fig. 4.

Joshi and Tsai in [18] proposed a method to determine a screw based Jacobian using the theory of reciprocal screws for a 


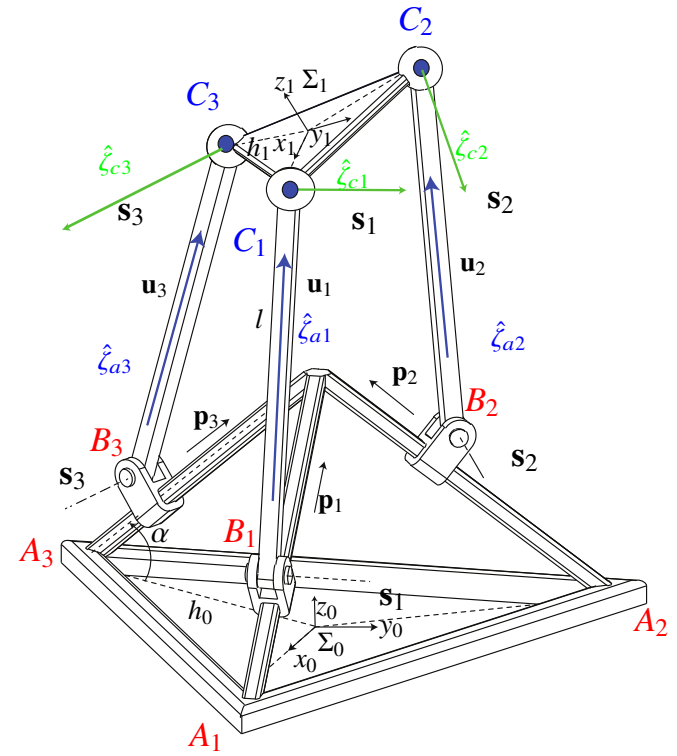

FIGURE 3: ACTUATION AND CONSTRAINT WRENCHES

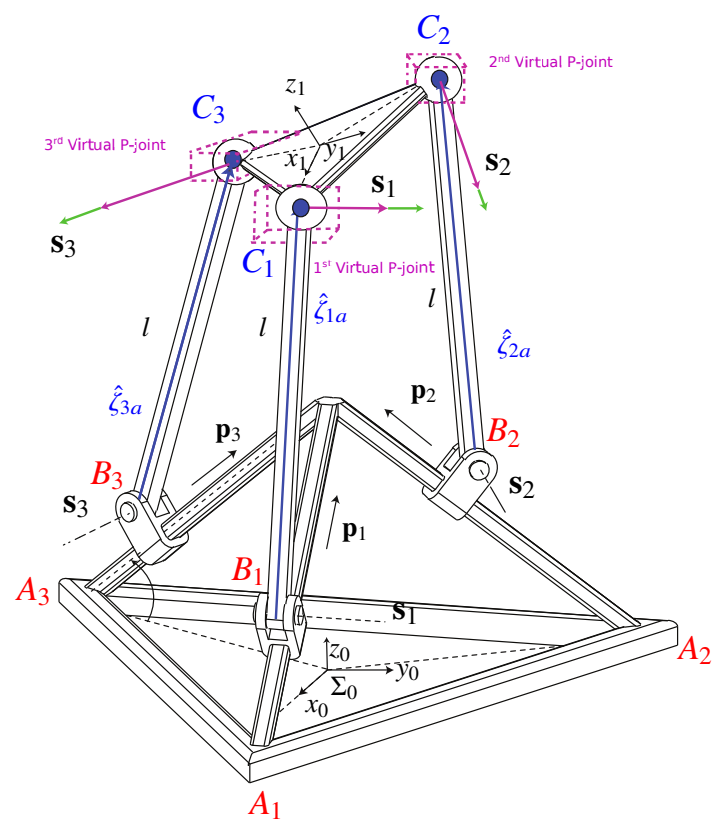

FIGURE 4: 3-PRS PM WITH VIRTUAL JOINTS

lower-mobility parallel manipulator. The twists associated with the prismatic joint $\left(\hat{\epsilon}_{\infty} 1_{i}\right)$, the revolute joint $\left(\hat{\epsilon}_{02_{i}}\right)$, the spherical joint $\left(\hat{\epsilon}_{03_{i}}, \hat{\epsilon}_{04_{i}}, \hat{\epsilon}_{05_{i}}\right)$ and the virtual prismatic joint $\left(\hat{\epsilon}_{\infty 6_{i}}\right)$ of the $i$-th limb can be expressed as:

$$
\begin{gathered}
\hat{\epsilon}_{\infty 1_{i}}=\left(\mathbf{0}_{3 \times 1}, \mathbf{p}_{i}\right), \hat{\epsilon}_{02_{i}}=\left(\mathbf{s}_{i}, \mathbf{r}_{C_{i}} \times \mathbf{s}_{i}\right), \hat{\epsilon}_{03_{i}}=\left(\boldsymbol{x}_{1}, \mathbf{r}_{C_{i}} \times \boldsymbol{x}_{1}\right), \\
\hat{\epsilon}_{04_{i}}=\left(\boldsymbol{y}_{1}, \mathbf{r}_{C_{i}} \times \boldsymbol{y}_{1}\right), \hat{\epsilon}_{05_{i}}=\left(z_{1}, \mathbf{r}_{C_{i}} \times z_{1}\right), \hat{\epsilon}_{\infty 6_{i}}=\left(\mathbf{0}_{3 \times 1}, \mathbf{s}_{i}\right)
\end{gathered}
$$

As all the five joints are linked as a serial chain, we may consider the $i$-th limb as a serial chain connecting the base to the moving platform. Therefore, we may represent the instantaneous twist of the moving platform as a linear combination of the twists of the joints of the corresponding serial chain, as follows:

$\mathbf{t}_{P}=\dot{\rho}_{1_{i}} \hat{\epsilon}_{\infty 1_{i}}+\dot{\theta}_{2_{i}} \hat{\epsilon}_{02_{i}}+\dot{\theta}_{3_{i}} \hat{\epsilon}_{03_{i}}+\dot{\theta}_{4_{i}} \hat{\epsilon}_{04_{i}}+\dot{\theta}_{5_{i}} \hat{\epsilon}_{05_{i}}+\dot{\rho}_{6_{i}} \hat{\epsilon}_{\infty 6_{i}}$

where, $\dot{\rho}$ and $\dot{\theta}$ are the respective joint rates. By making the reciprocal product on both sides of Eq. (11) with an actuation wrench $\hat{\zeta}_{a_{i}}$ of the $i$-th limb, it yields,

$$
\begin{gathered}
\hat{\zeta}_{a i}^{T} \circ \mathbf{t}_{p}=\dot{\rho}_{1_{i}} \hat{\zeta}_{a i}^{T} \hat{\epsilon}_{\infty 1_{i}} \\
\hat{\zeta}_{a i}^{T} \hat{\epsilon}_{\infty 1_{i}}=\left[\left(\mathbf{r}_{C_{i}}^{0} \times \mathbf{u}_{i}\right)^{T} \mathbf{u}_{i}^{T}\right]\left[\begin{array}{c}
\mathbf{0}_{3 \times 1} \\
\mathbf{p}_{i}
\end{array}\right]=\mathbf{u}_{i}^{T} \cdot \mathbf{p}_{i}
\end{gathered}
$$

Thus,

$$
\hat{\zeta}_{a i}^{T} \circ \mathbf{t}_{P}=\dot{\rho}_{1_{i}} \mathbf{u}_{i}^{T} \cdot \mathbf{p}_{i}
$$

By making the reciprocal product on both sides of Eq. (11) with a constraint wrench $\hat{\zeta}_{c i}$ of the $i$-th limb, it becomes,

$$
\begin{gathered}
\hat{\zeta}_{c i}^{T} \circ \mathbf{t}_{P}=\dot{\rho}_{6_{i}} \hat{\zeta}_{c i}^{T} \hat{\epsilon}_{\infty 6_{i}} \\
\hat{\zeta}_{c i}^{T} \hat{\epsilon}_{\infty 6_{i}}=\left[\left(\mathbf{r}_{C_{i}}^{0} \times \mathbf{s}_{i}\right)^{T} \mathbf{s}_{i}^{T}\right]\left[\begin{array}{c}
\mathbf{0}_{3 \times 1} \\
\boldsymbol{s}_{i}
\end{array}\right]=\mathbf{s}_{i}^{T} \cdot \mathbf{s}_{i}
\end{gathered}
$$

Thus,

$$
\hat{\zeta}_{c i}^{T} \circ \mathbf{t}_{P}=\dot{\rho}_{6_{i}}
$$

Writing Eqs. (12) and (13) for the three limbs of the manipulator, we get,

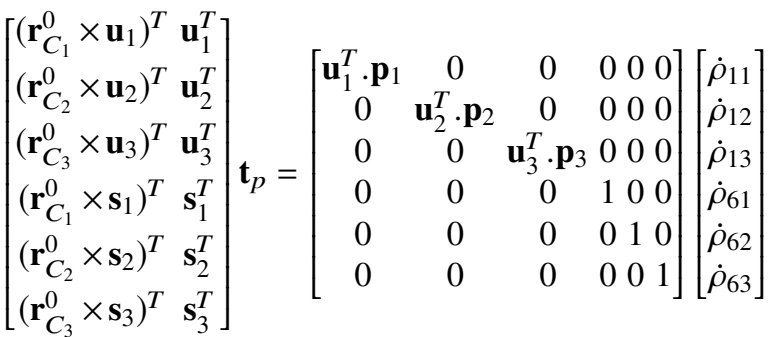

$$
\begin{aligned}
& \mathbf{A} \mathbf{t}_{P}=\mathbf{B} \rho \\
& \mathbf{t}_{P}=\mathbf{J}^{\prime} \rho
\end{aligned}
$$


Thus, Eq. (14) gives a $(6 \times 6)$ inverse Jacobian. The columns of this inverse Jacobian matrix are nothing but the output and constraint twists.

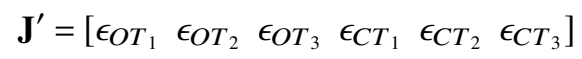

The screws of this matrix are normalized to obtain the Jacobian of unit output and constraint twists as follows:

$$
\mathbf{J}=\left[\begin{array}{llll}
\hat{\epsilon}_{O T_{1}} & \hat{\epsilon}_{O T_{2}} \hat{\epsilon}_{O T_{3}} \hat{\epsilon}_{C T_{1}} \hat{\epsilon}_{C T_{2}} \hat{\epsilon}_{C T_{3}}
\end{array}\right]
$$

where, $\hat{\epsilon}_{O T_{i}}=\frac{\epsilon_{O T_{i}}}{\lambda_{O T_{i}}}, \hat{\epsilon}_{C T_{j}}=\frac{\epsilon_{C T_{j}}}{\lambda_{C T_{j}}}$ and $\lambda_{O T_{i}}, \lambda_{C T_{j}}$ are the magnitudes of the output twist and constraint twists respectively.

\section{PERFORMANCE COMPARISON OF THE OPERATION MODES $x_{0}=0$ AND $x_{3}=0$}

In this section, the performance analysis of the two operation modes, i.e., $x_{0}=0$ and $x_{3}=0$, based on the results obtained for OTI and CTI is presented. Five different designs of the 3-PRS parallel manipulator are selected with $\alpha=0,30,45,60$ and 90 degrees. While the other design parameters are assigned as: $l=$ $3 m, h_{0}=2 m$, and $h_{1}=1 \mathrm{~m}$. Polar plots are generated to visualize the distribution of the output and constraint transmission indices in the orientation space of the manipulator, which is parametrized by the azimuth angle $\phi$ and tilt angle $\theta$, at constant height $Z$. Due to page limit, plots for only two $\alpha$ values, 0 and 30 degrees, are presented here. (In all the figure captions, 'OM' indicates Operation Mode.)

The distribution of output transmission index in operation mode $x_{0}=0$ can be seen from Figs. 5- 6 . At zero tilt and azimuth, output transmission index has a non zero value. It appears that the higher the tilt angle, the lower the OTI. In Fig. 5, the region where OTI has values less than 0.1 is a region where transmission of actuation forces is poor. It means that the moving-platform of the manipulator is poorly controlled in this area and the manipulator is close to an actuation singularity. The regions of poor transmissions are quite consistent with the singularity loci determined in [1]. For operation mode $x_{3}=0$, Figs. 7-8 show a significant difference in the distribution of OTI values as compared with operation mode $x_{0}=0$. Therefore, it can be seen that the higher the angle $\alpha$, the larger the orientation workspace of the 3-PRS parallel manipulator free of actuation singularity in both operation modes.

Figures 9 and 10 show the distribution of constraint transmission index for $\alpha$ equal to 0 and 30 degrees in operation mode $x_{0}=0$. For $\alpha=0^{0}$, at zero tilt and azimuth, CTI has very high value (around 0.9). This implies optimal transmission of constraint forces. The value stays higher over a certain range of tilt and then drops down indicating poor transmission of constraint forces. This implies poor constraining abilities and hence poor positional accuracy. For operation mode $x_{3}=0$, a significant dif-

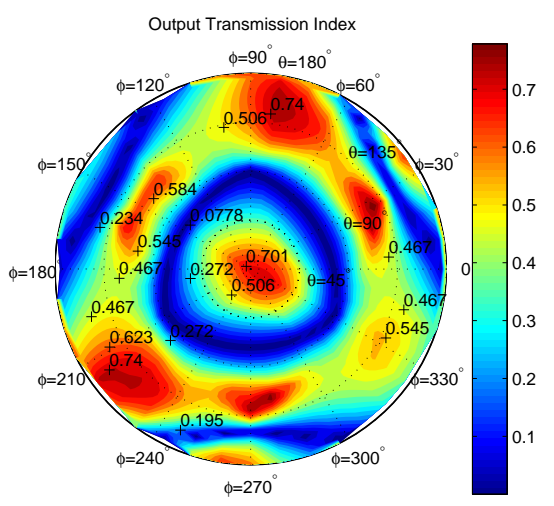

FIGURE 5: OM1: OTI $\left(\alpha=0^{0}, Z=2 \mathrm{~m}\right)$

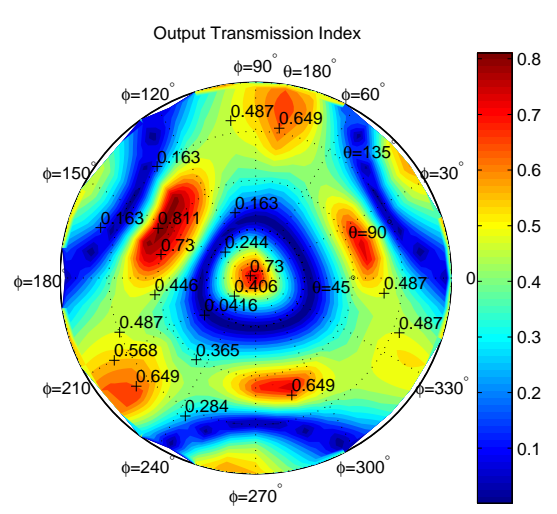

FIGURE 6: OM1: OTI $\left(\alpha=30^{\circ}, Z=2 \mathrm{~m}\right)$

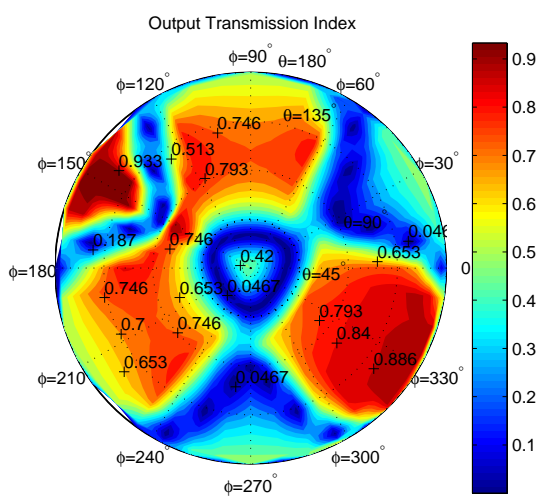

FIGURE 7: OM2: OTI $\left(\alpha=0^{0}, Z=2 \mathrm{~m}\right)$ 


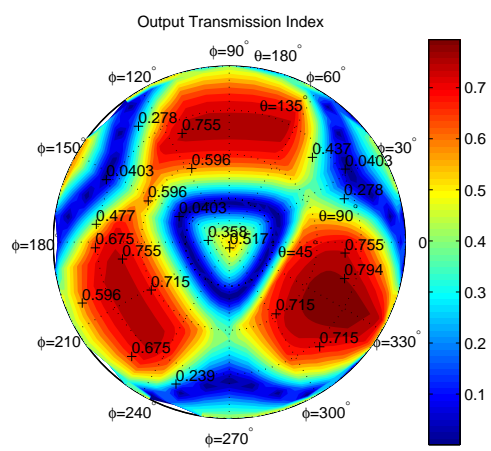

FIGURE 8: OM2: OTI $\left(\alpha=30^{\circ}, Z=2 \mathrm{~m}\right)$

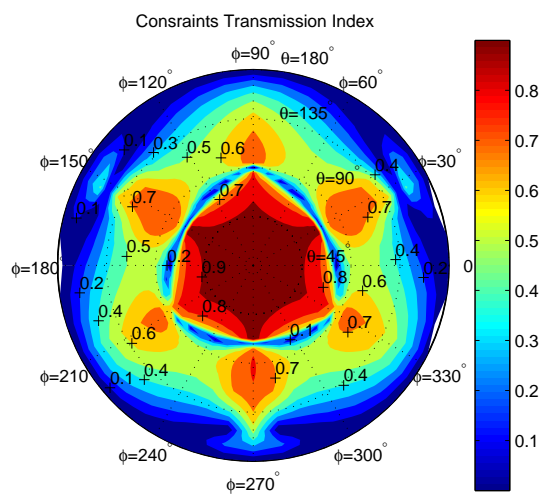

FIGURE 9: OM1: CTI $\left(\alpha=0^{0}, Z=2 \mathrm{~m}\right)$

ference in the distribution of CTI values can be seen from Figs. 11-12 as compared to operation mode $x_{0}=0$. Therefore, it reveals that the constrainability of the manipulator decreases with the increment of parameter angle $\alpha$ in operation mode $x_{0}=0$. Whereas in operation mode $x_{3}=0$, the constrainability of the manipulator increases with the increment of parameter angle $\alpha$.

For better comparison, 2-D curves of both the indices are generated for different values of angle $\alpha$ with respect to tilt angle $\theta$, at constant azimuth angle $\phi$. By comparing Figs. 13 and 14, we can see that, at zero tilt, in operation mode $x_{0}=0$, the higher the angle $\alpha$, the lower the OTI. However, in operation mode $x_{3}=$ 0 , at zero tilt, the higher the angle $\alpha$, the higher the OTIs. Some differences in the orientation workspace for the two operation modes can also be seen. In operation mode $x_{0}=0$, the higher the angle $\alpha$, the lower the orientation workspace free of singularity, while in operation mode $x_{3}=0$, the higher the angle $\alpha$, the higher the orientation workspace free of singularity. In terms of force transmission efficiency, operation mode $x_{0}=0$ is better than $x_{3}=$ 0 .

From Figs.15 and 16 we can see that, at zero tilt, in opera-

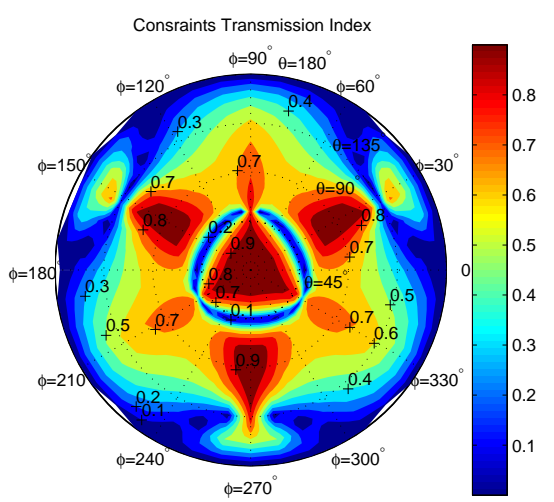

FIGURE 10: OM1: CTI $\left(\alpha=30^{\circ}, Z=2 \mathrm{~m}\right)$

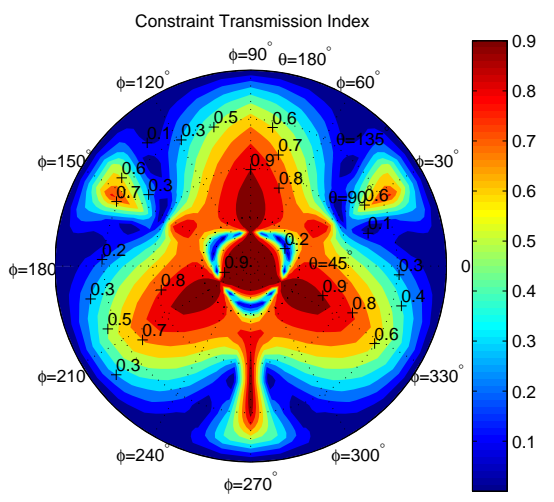

FIGURE 11: OM2: CTI $\left(\alpha=0^{0}, Z=2 \mathrm{~m}\right)$

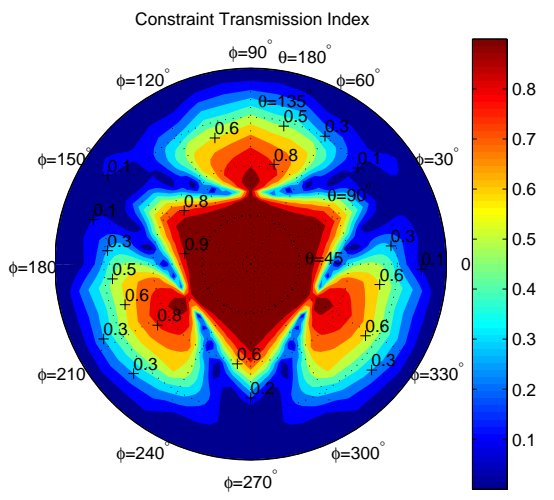

FIGURE 12: OM2: CTI $\left(\alpha=30^{\circ}, Z=2 \mathrm{~m}\right)$ 


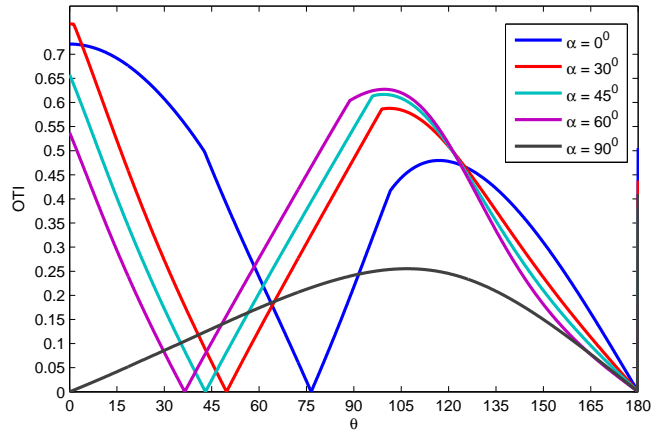

FIGURE 13: OM1: OTI Curves at $\phi=0^{0}$ and $Z=2 \mathrm{~m}$

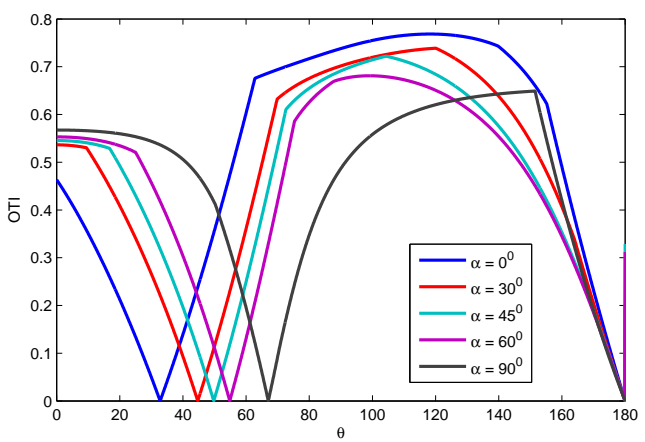

FIGURE 14: OM2: OTI Curves at $\phi=0^{0}$ and $Z=2 \mathrm{~m}$

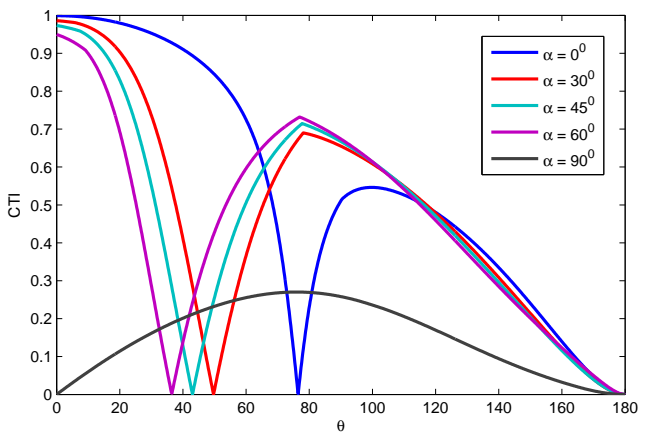

FIGURE 15: OM1: CTI Curves at $\phi=0^{0}$ and $Z=2 \mathrm{~m}$

tion mode $x_{0}=0$, CTI values show decreasing trend for increase in the value of $\alpha$. However, in operation mode $x_{3}=0$, at zero tilt, CTI is optimal for all $\alpha$ values. From Fig.13 and 15, the manipulator can be seen in singular configuration at zero tilt for $\alpha=90^{\circ}$. Thereafter, the manipulator reaches singularity only at $\theta=180^{\circ}$ degrees. In contrast to OTI, CTI curves show higher values near zero tilt. This implies higher constrainability and hence higher positional accuracy.

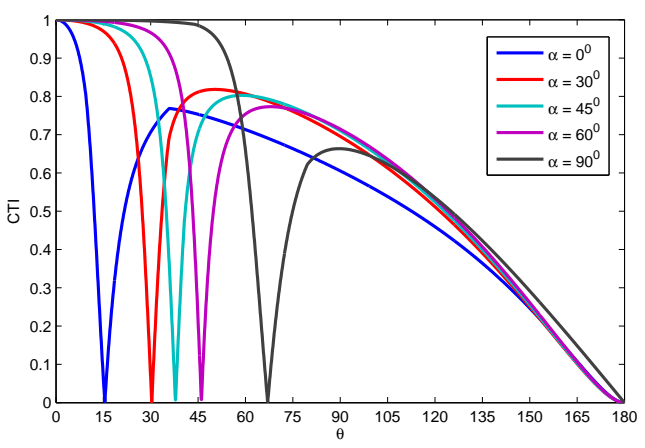

FIGURE 16: OM2: CTI Curves at $\phi=0^{0}$ and $Z=2 \mathrm{~m}$

\section{SINGULARITY CONFIGURATIONS IN OPERATION MODES $x_{0}=0$ AND $x_{3}=0$}

OTI and CTI are supposed to return a zero value when the manipulator reaches an actuation or a constraint singularity [17]. Thus, manipulator plots are obtained at zero values of the indices to realize the pose of the manipulator. As the output transmission index determines the amount of actuation force transmitted to the manipulator's platform, a zero value of this index was claimed to indicate an actuation singularity. However, it turns out that in singular configurations shown in Figs. 17 and 19, a screw $\hat{\zeta}_{r}$ which is simultaneously reciprocal to all the three actuation wrenches, is also reciprocal to the three constraint wrenches of the manipulator. Thus, these configurations, in operation mode $x_{0}=0$, actually represent a case of compound singularity, as introduced in [19]. Compound singularities in operation mode $x_{3}=0$ are shown in Figs. 21 and 23. Likewise, a zero value of constraint transmission index was claimed to indicate constraint singularity. Figures 18, 20, 22 and 24 indicate constraint singularities. In these configurations, all the three constraint wrenches (shown in figures by green coloured vectors $\mathbf{s}_{1}, \mathbf{s}_{2}$, and $\mathbf{s}_{3}$ ) intersect at a common point. Thus, manipulator gains an extra $d o f$. The axis of this extra gained motion intersects the three constraint wrenches and is perpendicular to them.

\section{CONCLUSIONS}

In this paper, the two operation modes of a reconfigurable 3-PRS parallel manipulator were compared based on output and constraint transmission indices. The kinematic performance of the manipulator is a function of the angle $\alpha$ that parametrizes its reconfigurability.

By calculating the output transmission index, the study of actuation force transmission capabilities of the two operation modes was carried out. For this purpose, five different designs of the 3-PRS parallel manipulator, associated with $\alpha=0,30,45$, 60, 90 degrees, were considered. It was observed that, the two operation modes differ in terms of transmitting actuation forces at given values of design parameters. The effect of angle $\alpha$ on the 


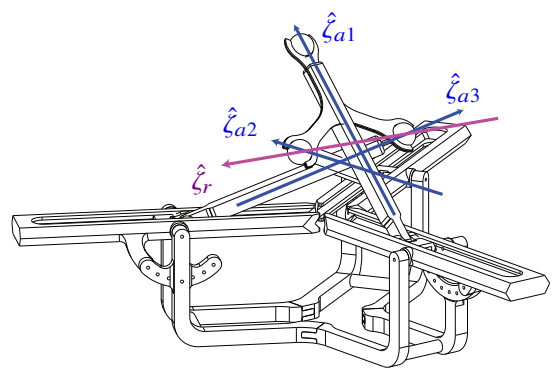

FIGURE 17: OM1: Compound Singularity at $\alpha=0^{0}$

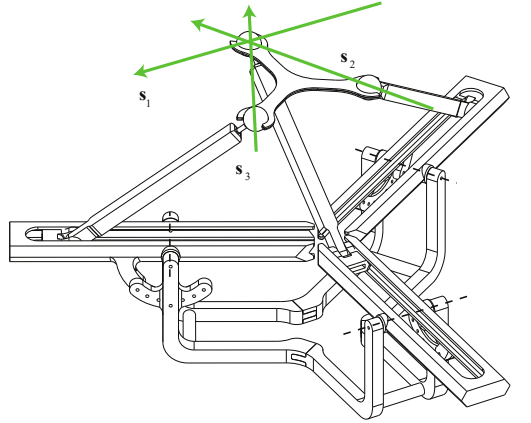

FIGURE 18: OM1: Constraint Singularity at $\alpha=0^{0}$

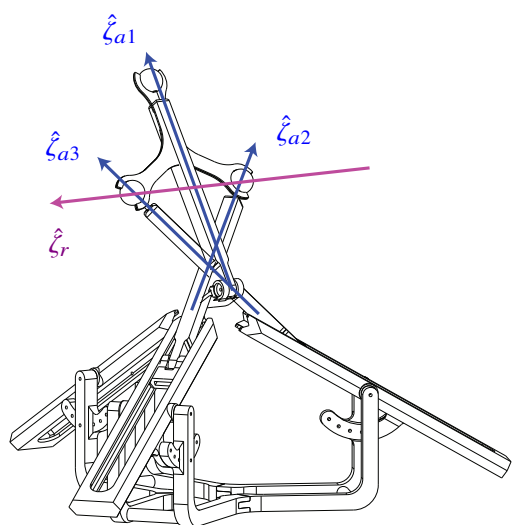

FIGURE 19: OM1: Compound Singularity at $\alpha=30^{\circ}$

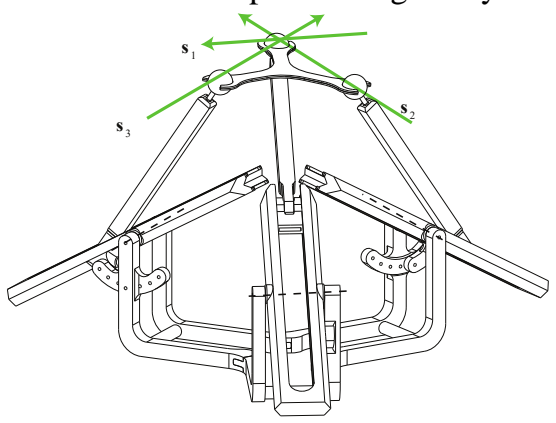

FIGURE 20: OM1: Constraint Singularity at $\alpha=30^{\circ}$

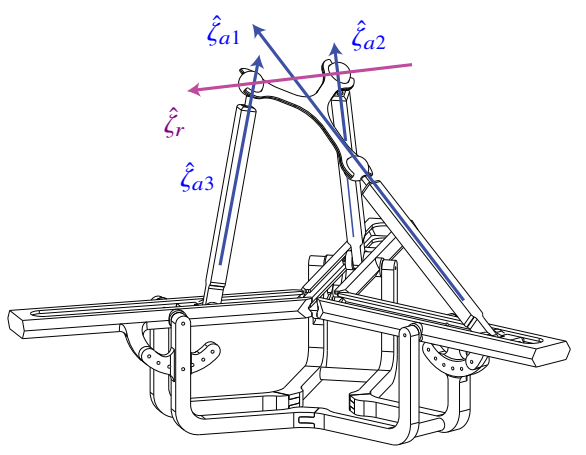

FIGURE 21: OM2: Compound Singularity at $\alpha=0^{0}$

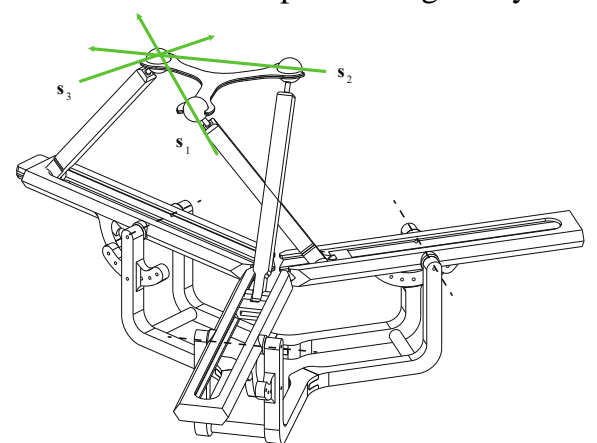

FIGURE 22: OM2: Constraint Singularity at $\alpha=0^{0}$

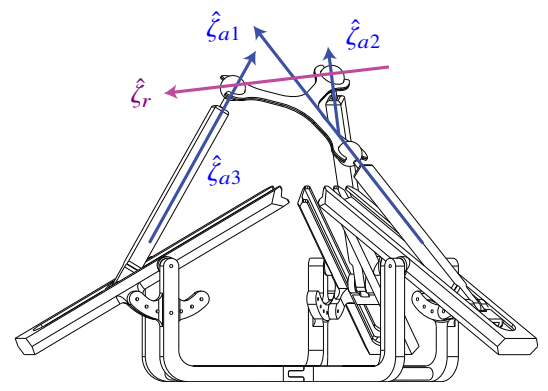

FIGURE 23: OM2: Compound Singularity at $\alpha=30^{0}$

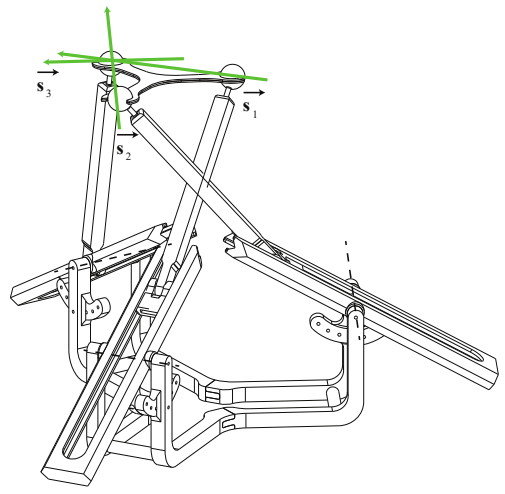

FIGURE 24: OM2: Constraint Singularity at $\alpha=30^{\circ}$ 
force transmission capabilities and orientation workspace was observed for both the operation modes of the manipulator. It is noteworthy that the higher the $\alpha$, the lower the orientation workspace free of singularity in operation mode $x_{0}=0$. Constraint transmission index was also evaluated to study the constraining abilities of the manipulator. It reveals that the manipulator designs have higher values of constraint transmission index than OTI and hence has a good positional accuracy. Operation mode $x_{0}=0$ was found to perform higher force transmission than mode $x_{3}=0$ at zero tilt.

OTI and CTI were claimed to predict singularities. Investigation of this claim was another motivation of this paper. OTI and CTI simultaneously yield zero values making it difficult to differentiate between actuation and constraint singularities. Thus, the investigation of the condition of OTI and CTI vanishing simultaneously will be the subject of future work.

\section{REFERENCES}

[1] Nurahmi, L., Caro, S., and Wenger, P., 2015, “Operation Modes and Singularity analysis of 3-PRS Parallel Manipulators with different arrangements of P-joints", Proceedings of the ASME 2015 International Design Engineering Technical Conference and Computers and Information in Engineering Conference, Boston, Massachusetts, USA

[2] Nurahmi, L., Caro, S., Wenger, P., Husty, M., and Schadlbauer, J., 2016,"Reconfiguration Analysis of a 4-RUU Parallel Manipulator", Mechanism and Machine Theory, Vol 96, Part 2, pp. 269-289.

[3] Li, Y. and Xu, Q., 2007, "Kinematic analysis of a 3-PRS parallel manipulator", Robotics and Computer-Integrated Manufacturing, 23, pp. 395 - 408.

[4] Wahl, J., 2000, "Articulated Tool Head", WIPO Patent, No. WO 00/25976.

[5] Liu, X-J., and Bonev, I., 2008, “Orientation Capability, Error Analysis, and Dimensional Optimization of Two Articulated Tool Heads With Parallel Kinematics", Journal of Manufacturing Science and Engineering, 130, pp. 011015-1-011015-9.

[6] Fan, K-C., Wang, Hai., Zhao, J-W., and Chang, T-H., 2003, "Sensitivity analysis of the 3-PRS parallel kinematic spindle platform of a serial-parallel machine tool," International Journal of Machine Tools and Manufacture, 43(15), pp. 1561-1569.

[7] D. M. Gan, J. S. Dai, Jorge Dias, and L. D. Seneviratne, "Variable Motion/Force Transmissibility of a Metamorphic Parallel Mechanism with Reconfigurable 3T and 3R Motion", Transactions of the ASME: Journal of Mechanisms and Robotics, 2016, doi:10.1115/1.4032409.

[8] D. M. Gan, Jorge Dias, and L. D. Seneviratne, "Unified Kinematics and Optimal Design of a 3rRPS Metamorphic Parallel Mechanism with a Reconfigurable Revolute
Joint", Mechanism and Machine Theory, 2016, 96 (part 2), pp. 239-254.

[9] Nurahmi, L., Schadlbauer, J., Caro, S., Husty, M., and Wenger, P., 2015, "Kinematic Analysis of of the 3-RPS Cube Parallel Manipulator", ASME Journal of Mechanisms and Robotics, Vol 7, Issue 1, pp. 011008-1-01100810.

[10] Ball, R.S, 1900, "A treatise on the theory of screws," Cambridge University Press, Cambridge,

[11] Sutherland, G., and Roth, B., 1973, "A transmission Index for spatial mechanisms," ASME journal of Engineering for industry, pp. 589-597

[12] Chen, C. and Angeles, J., 2007,"Generalized transmission index and transmission quality for spatial linkages", Mech. Mach. Theory, 42(9) pp.1225-1237

[13] Liu, X-J., Wang, L-P., Xie, F. and Bonev, I., 2010, "Design of a Three-Axis Articulated Tool Head With Parallel Kinematics Achieving Desired Motion/Force Transmission Characteristics," Journal of Manufacturing Science and Engineering, 132, pp. 021009-1-021009-8.

[14] Wang, J., Wu, C. and Liu, X-J., 2010, "Performance Evaluation of Parallel Manipulators: Motion/Force Transmissibility and Its Index", Mechanism and Machine Theory, 45(10), pp. 1462-1476

[15] Liu, X-J., Wu, C. and Wang, J., 2012, "A New Approach for the Singularity Analysis and Closeness Measurement to Singularities of Parallel Manipulators," ASME Journal of Mechanisms and Robotics, 4(4), pp.041001-1-041001-10

[16] Liu, X-J., Chen, X. and Nahon, M., 2014, "Motion/Force Constrainability Analysis of Lower-Mobility Parallel Manipulators," ASME Journal of Mechanisms and Robotics, 6(3) pp.031006-1-031006-9

[17] Liu, H., Huang. T., Kecskemethy, A. and Chetwynd, D. G., 2014, "A generalized approach for computing the transmission index of parallel mechanisms," Mechanism and Machine Theory, 74, pp. 245-256

[18] Joshi, S., Tsai, L-W., 2002, "Jacobian Analysis of Limited-DOF Parallel Manipulators," Journal of Mechanical Design, 124(2), pp. 254-258

[19] Nurahmi, L., and Caro, S., 2015, "Dimensionally Homogeneous Jacobian and Condition Number", 2nd International Conference on Mechanical Engineering, Denpasar, Bali, Indonesia. 